\title{
Iron Abundances of the Cluster RR Lyrae Variables Based on Their Light Curves
}

\author{
Johanna Jurcsik and Géza Kovács \\ Konkoly Observatory, H-1525 Budapest XII, Box 67, Hungary
}

\begin{abstract}
Fe} / \mathrm{H}]$ values of cluster RRab variables were calculated from the Fourier parameters of their light curves using a recently published method. The average cluster metallicities obtained this way agree with direct observations within the error range.
\end{abstract}

\section{Introduction}

A previously obtained light curve $\rightarrow[\mathrm{Fe} / \mathrm{H}]$ calibration (Kovács \& Zsoldos, 1995) was applied on independent samples of cluster variables. Good quality CCD, photoelectric and some photographic observations were used for 8 galactic and $5 \mathrm{LMC}$ clusters. Iron abundances of $83 \mathrm{RRab}$ stars were determined according to the 3 and 5 parameter formulae which relate the Fourier parameters of the $V$ light curves to metallicity.

Because the calibrating formulae were constructed by use of $[\mathrm{Fe} / \mathrm{H}]$ values which were obtained from $\Delta S$ observations through Blanco's transformation (Blanco, 1992), our results are compared with the $\Delta S$ cluster abundances on this scale. For clusters with no $\Delta S$ measurements the metallicities derived from different methods (mostly high dispersion spectroscopy) were also transformed to the Blanco scale.

$[\mathrm{Fe} / \mathrm{H}]$ values with error less than $0.5 \mathrm{dex}$ are regarded as reliable. The smallest error of the individual $[\mathrm{Fe} / \mathrm{H}]$ determinations is about 0.04 dex.

\section{Results}

Our results are shown in Figure 1. The following conclusions can be drawn.

- For less metal deficient clusters the calculated $[\mathrm{Fe} / \mathrm{H}]$ values are in good agreement with the average cluster abundances.

- For the most metal deficient clusters our values are somewhat high, probably because of the small number of the very metal poor stars in our sample of calibration.

- The internal standard deviations of $[\mathrm{Fe} / \mathrm{H}]$ for the individual clusters vary between 0.06-0.23 dex with a bias towards higher standard deviations for the lower quality light curves. The only exception is the LMC cluster NGC 2257 where the individual abundances suggest chemical inhomogeneity or more probably non-membership of some of the variables. The spectroscopic abundance determinations give two distinct values of the metallicity of this cluster. The 


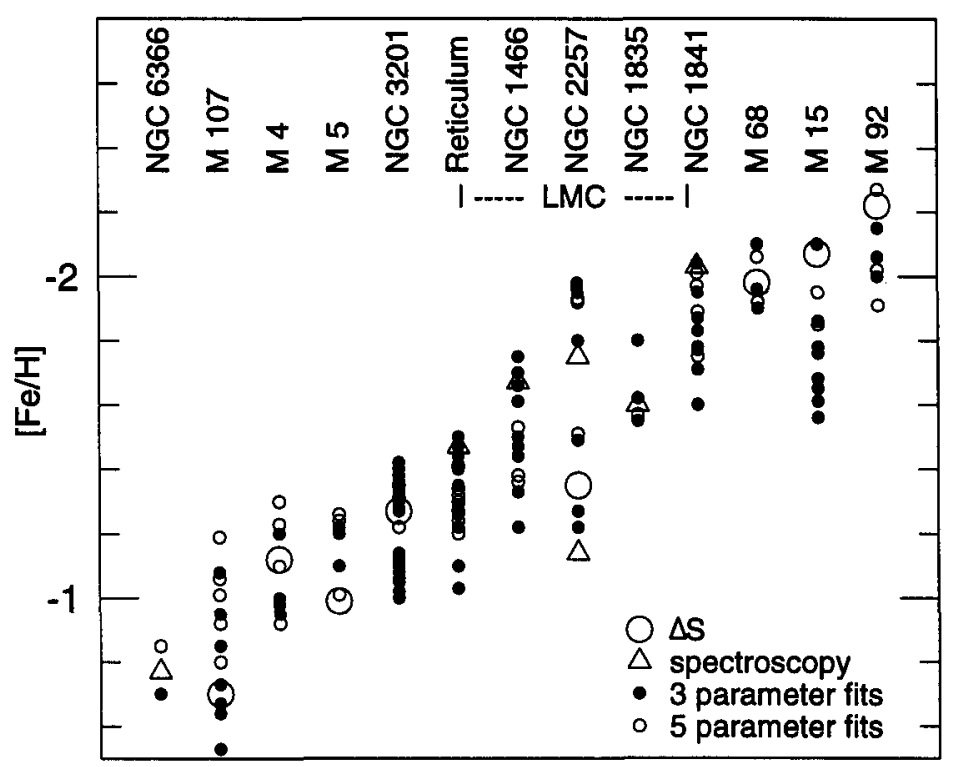

Figure 1. $\quad[\mathrm{Fe} / \mathrm{H}]$ values of individual RRab stars calculated from the Fourier parameters of their light curves $(\circ, \bullet)$. The average cluster metallicities $(\Delta S(O)$ or spectroscopy $(\Delta)$, transformed to the Blanco scale) are shown for comparison.

less metal poor solution is, however, close to the $\Delta S$ abundance of the surrounding field RR Lyrae stars. Therefore, we conclude that the variables with $[\mathrm{Fe} / \mathrm{H}]$ values similar to this higher metal abundance could be field variables, too.

- The present method is not applicable for the estimation of $[\mathrm{Fe} / \mathrm{H}]$ in peculiar cases (e.g., in Blazhko variables, highly evolved stars...). The $[\mathrm{Fe} / \mathrm{H}]$ values computed for stars with variable light curves are usually deviant. The anomalous $[\mathrm{Fe} / \mathrm{H}]$ we got for M4-V2, M68-V22, NGC 1841-V6 and 47 Tuc-V9 indicate these types of peculiarities and therefore they are not included in our sample.

- The accuracy of the transformation formulae could be improved by the use of larger number of the calibrating stars with more accurate $[\mathrm{Fe} / \mathrm{H}]$ values and light curves. This would enable us to derive precise metallicities on a large sample of RR Lyrae stars without resorting to the more tedious spectroscopic observations.

Acknowledgments. The financial support of OTKA (grant T-014183) is gratefully acknowledged.

\section{References}

Kovács, G., \& Zsoldos, E. 1995, A\&A, 293, L57

Blanco, V.M. 1992, AJ, 104, 734 